\title{
Quantum Zeno effect with partial measurement and noisy dynamics
}

\author{
Parveen Kumar $\odot,{ }^{1}$ Alessandro Romito, ${ }^{2}$ and Kyrylo Snizhko $\oplus^{1}$ \\ ${ }^{1}$ Department of Condensed Matter Physics, Weizmann Institute of Science, Rehovot 76100, Israel \\ ${ }^{2}$ Department of Physics, Lancaster University, Lancaster LA1 4YB, United Kingdom
}

(Received 26 June 2020; accepted 10 December 2020; published 24 December 2020)

\begin{abstract}
We study the quantum Zeno effect (QZE) induced by continuous partial measurement in the presence of short-correlated noise in the system Hamiltonian. We study the survival probability and the onset of the QZE as a function of the measurement strength and find that, depending on the noise parameters, the quantum Zeno effect can be enhanced or suppressed by the noise in different regions of parameter space. Notably, the conditions for the enhancement of the QZE are different when determined by the short-time or long-time behavior of the survival probability, or by the measurement strength marking the onset of the quantum Zeno regime.
\end{abstract}

DOI: 10.1103/PhysRevResearch.2.043420

\section{INTRODUCTION}

The evolution of a quantum system can be "frozen" by repeated projective measurements; this phenomenon is known as the quantum Zeno effect (QZE) [1-3]. The QZE originates from the general feature of Schrödinger evolution: at short times, transition probabilities between quantum states are quadratic in time; hence if a system in a measurement eigenstate is measured at regular intervals $\Delta t$, the probability of a transition to another state is asymptotically small in the limit $\Delta t \rightarrow 0[4,5]$. The QZE is a well established experimental reality: it has been explored experimentally in various setups such as trapped ions [2], polarized photons [6], cold atoms [7], dilute Bose-Einstein condensed gases [8], nanomechanical oscillators [9], and superconducting qubits [10]. Besides confining a system in a specific state, the QZE can be used for a few other purposes such as stabilizing a multidimensional subspace $[3,11]$ (a feature which has been experimentally observed with a rubidium Bose-Einstein condensate [12,13] and that can be useful for quantum error correction [14-18]) or tuning the degree of Markovianity in the dynamics of an open quantum system [19]. Furthermore, the QZE can play an important role in the dynamics of many-body systems [20,21].

Given the potential applications of the QZE, it is important to study the effect under realistic nonideal conditions, in which uncertainties in the system Hamiltonian and in the measurement process are unavoidable. For example, recent studies addressed the QZE due to randomly spaced projective measurements [22,23] or under nonprojective (generalized) measurements [8,17,24-30]. Interestingly, it has been found that a complete stabilization of the system in specific states is

Published by the American Physical Society under the terms of the Creative Commons Attribution 4.0 International license. Further distribution of this work must maintain attribution to the author(s) and the published article's title, journal citation, and DOI. possible even with imperfect measurements occurring at finite frequencies [29].

In this work, we study a different kind of nonideality. While the above-mentioned works assumed a fully known and controlled Hamiltonian, we consider a system with a noisy Hamiltonian subjected to continuous partial measurements [31-35], cf. Fig. 1. Several specific types of noise have been previously considered [36-39]. In particular, it has been shown that, in the presence of short-correlated noise, the system dynamics cannot be frozen completely [39]. Nevertheless, the presence of measurement can slow down the system's departure from the desired state. We investigate whether the QZE (in the sense of slowing the system decay down) is enhanced or suppressed by an arbitrary short-correlated noise in the Hamiltonian as compared with the case in which the noise is absent. We use three distinct observables to address the effect of noise in this regard: the short-time and the longtime survival probabilities, as well as the critical value of the measurement strength for the onset of the QZE. The latter is identified via a transition from the regime in which population of the state of interest oscillates in time to the regime in which it monotonically decays (with the decay rate vanishing for the perfect QZE) [40,41]. We find that the noise can enhance the QZE. At the same time, the conditions for enhancing the QZE behavior differ drastically for each of the observables. While the short-time probability of staying ("surviving") in the initial state is essentially always reduced by noise, the long-time survival probability is increased in some parameter range, specifically when the measurement strength and the average Hamiltonian parameters fall within a certain noisedetermined region. Finally, we identify a set of constraints for the noise strength and average Hamiltonian parameters which extend the QZE regime by inducing the transition to the QZE at a smaller measurement strength. Notably, these constraints, do not exactly match those determining the region of the long-time survival probability enhancement.

This paper is organized as follows: Section II introduces the setup under consideration and its modeling. The features of the QZE in our setup when the Hamiltonian is noiseless 


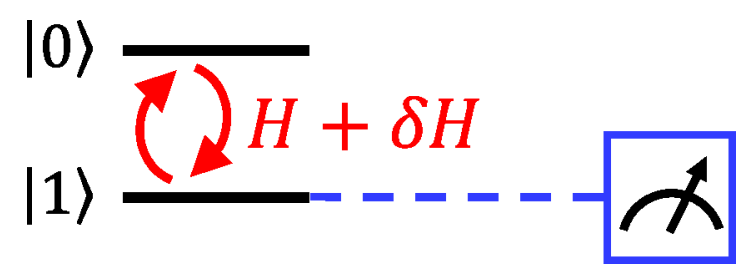

FIG. 1. The setup under consideration. A two-level system is subject to a fluctuating Hamiltonian that induces quantum oscillations between the two levels. Simultaneously, the system is subject to a continuous partial measurement: the detector has a finite probability per unit time to click when the system is in state $|1\rangle$, making the detection of the system state imperfect.

are reviewed in Sec. III. In Sec. IV, we investigate the effect of noise on the QZE. We conclude with a discussion of our results in Sec. V.

\section{THE SETUP}

We consider a two-level system, whose basis states are labeled $|0\rangle$ and $|1\rangle$, see Fig. 1. We represent the system state by the density matrix

$$
\rho_{s}(t)=\frac{1}{2}(\mathbb{I}+\boldsymbol{s} \cdot \boldsymbol{\sigma}),
$$

where $s=\{x(t), y(t), z(t)\}$ is the Bloch vector, $\mathbb{I}$ is the identity operator, and $\sigma=\left\{\sigma_{x}, \sigma_{y}, \sigma_{z}\right\}$ are the Pauli spin operators defined by $\sigma_{z}|0\rangle=|0\rangle$. We consider the system's initial state to be

$$
\rho_{s}(0)=|0\rangle\langle 0|=\left(\begin{array}{ll}
1 & 0 \\
0 & 0
\end{array}\right) .
$$

The system evolution is dictated by the interplay of two ingredients: a Hamiltonian including an explicit noise component and the backaction of the measurements. We describe their effect separately, in Secs. II A and II B, before combining them to derive the full master equation for the system's density matrix in Sec. II C.

\section{A. System Hamiltonian and noise}

In the absence of measurements, the system undergoes a unitary evolution with the Hamiltonian

$$
H(t)=\omega \sigma_{x}+\boldsymbol{\xi}(t) \cdot \sigma,
$$

where $\omega$ drives Rabi oscillations between the two levels, and $\boldsymbol{\xi}(t)$ is a time-dependent short-correlated noise with a Gaussian distribution such that $\left\langle\xi_{i}(t)\right\rangle=0$ and $\left\langle\xi_{i}(t) \xi_{j}\left(t^{\prime}\right)\right\rangle=$ $\gamma_{i j} \delta\left(t-t^{\prime}\right)$, where $i \in\{x, y, z\}$. Here $\gamma_{i j}$ is a real positive semidefinite matrix and $\delta(t)$ is the Dirac delta function. In what follows, we discretize the evolution into time steps of size $d t$, in which case $\delta\left(t-t^{\prime}\right)=\delta_{t, t^{\prime}} / d t$, where $\delta_{t, t^{\prime}}$ is the Kronecker delta. Each individual realization of the noise generates a stochastic trajectory of the system state evolution on the Bloch sphere and the system state remains pure during the whole evolution $\left[s^{2}(t)=1\right]$. After averaging over different noise realizations, the system state becomes mixed, and the corresponding Bloch vector points to the interior of the Bloch sphere $\left[s^{2}(t)<1\right]$. In this work, we will be interested in the averaged evolution of the system state.
The system state evolution for one time step, averaged over the noise, is given by

$$
\begin{aligned}
\rho_{s}(t+d t)= & \left\langle U_{t} \rho_{s}(t) U_{t}^{\dagger}\right\rangle_{\xi(t)} \\
= & \rho_{s}(t)-i \omega\left[\sigma_{x}, \rho_{s}(t)\right] d t \\
& -\gamma_{i j}\left(\frac{1}{2}\left\{\sigma_{i} \sigma_{j}, \rho_{s}(t)\right\}-\sigma_{i} \rho_{s}(t) \sigma_{j}\right) d t+O\left(d t^{2}\right),
\end{aligned}
$$

where $U_{t}=e^{-i H(t) d t},[\ldots, \ldots]$ and $\{\ldots, \ldots\}$ stand for the commutator and the anticommutator, respectively.

\section{B. The measurement model}

The measurement consists of continuous partial measurements, see Fig. 1. Depending on the probability of detecting the system in the $|1\rangle$ state, the measurements can bridge between no measurement (vanishing backaction) and the projective measurement. Continuous partial measurements have been realized in various experimental architectures [16,42].

A simple physical model of partial measurements is obtained by considering a two-level-system detector (with basis states $\left.|0\rangle_{d},|1\rangle_{d}\right)$ initialized at the beginning of each measurement in state $|\psi\rangle_{d}=|0\rangle_{d}$. The joint system-detector state before the measurement can thus be written as

$$
\rho(t)=\rho_{s}(t) \otimes\left(|\psi\rangle_{d}\left\langle\left.\psi\right|_{d}\right) .\right.
$$

During the measurement, the system-detector interaction,

$$
H_{\text {int }}=\frac{J}{2}\left(\mathbb{I}-\sigma_{z}\right) \otimes \sigma_{y}^{(d)},
$$

is switched on for time interval $d t$; here $J$ determines the coupling strength between the system and the detector. The entangled system-detector state after the time step $d t$ is given by

$$
\rho(t+d t)=V \rho(t) V^{\dagger},
$$

where $V=e^{-i H_{\text {int }} d t}$. At the end, the detector state is measured projectively in the $\left\{|0\rangle_{d},|1\rangle_{d}\right\}$ basis. The measurement procedure is then repeated, giving rise to continuous partial measurement.

The system state after the measurement is obtained by tracing over the detector degrees of freedom,

$$
\rho_{s}(t+d t)=\operatorname{Tr}_{d}[\rho(t+d t)]=M_{0} \rho_{s}(t) M_{0}^{\dagger}+M_{1} \rho_{s}(t) M_{1}^{\dagger},
$$

where $M_{r}$ are the Kraus operators [43,44] encoding the measurement backaction corresponding to a particular readout $r=0,1$, i.e., when the detector is found to be in state $|r\rangle_{d}$ at the end of the measurement. They are given by $M_{r}=$ ${ }_{d}\langle r|V| 0\rangle_{d}$. Using Eq. (6), the Kraus operators $M_{0}$ and $M_{1}$ can be written as

$$
M_{0}=\left(\begin{array}{cc}
1 & 0 \\
0 & \cos (J d t)
\end{array}\right), \quad M_{1}=\left(\begin{array}{cc}
0 & 0 \\
0 & \sin (J d t)
\end{array}\right) .
$$

Equations (8) and (9) are the defining properties of the partial measurement used hereafter. They are independent of the specific detector model and could equivalently result from a measurement process different from the one used to illustrate their derivation. 
Note that the measurement strength is quantified by $J d t$ such that $J d t=0$ corresponds to no measurement, while $J d t=\pi / 2$ corresponds to strong (or projective) measurement. We obtain the continuum limit, $d t \rightarrow 0$, by scaling $J$ such that $J^{2} d t=$ const. $\equiv \alpha$. The master equation giving the evolution of the system density matrix under continuous partial measurement then follows as

$$
\begin{aligned}
\rho_{s}(t+d t)= & \rho_{s}(t)-\alpha\left(\frac{1}{2}\left\{P_{1}, \rho_{s}(t)\right\}\right. \\
& \left.-P_{1} \rho_{s}(t) P_{1}\right) d t+O\left(d t^{2}\right),
\end{aligned}
$$

where $P_{1}=|1\rangle\langle 1|=\left(\mathbb{I}-\sigma_{z}\right) / 2$.

\section{Combined system evolution}

The situation of interest for us is that the Hamiltonian, see Sec. II A, and the measurement-induced, see Sec. II B, evolutions happen simultaneously. In a single small time step $d t$, the two processes do not interfere with each other (up to order $d t$ ), so that

$$
\begin{aligned}
\rho_{s}(t+d t)= & \left\langle M_{0} U_{t} \rho_{s}(t) U_{t}^{\dagger} M_{0}^{\dagger}+M_{1} U_{t} \rho_{s}(t) U_{t}^{\dagger} M_{1}^{\dagger}\right\rangle_{\xi(t)}+O\left(d t^{2}\right) \\
= & \rho_{s}(t)-i \omega\left[\sigma_{x}, \rho_{s}(t)\right] d t \\
& -\gamma_{i j}\left(\frac{1}{2}\left\{\sigma_{i} \sigma_{j}, \rho_{s}(t)\right\}-\sigma_{i} \rho_{s}(t) \sigma_{j}\right) d t \\
& -\alpha\left(\frac{1}{2}\left\{P_{1}, \rho_{s}(t)\right\}-P_{1} \rho_{s}(t) P_{1}\right) d t+O\left(d t^{2}\right) .
\end{aligned}
$$

This master equation is equivalent to the following equation for the Bloch vector, cf. Eq. (1),

$$
\frac{d s}{d t}=\mathcal{L} s, \quad \mathcal{L}=\mathcal{L}_{0}+\mathcal{L}_{\gamma},
$$

where the evolution superoperator $\mathcal{L}$ (Liouvillian) is decomposed into the noiseless part

$$
\mathcal{L}_{0}=\left(\begin{array}{ccc}
-\frac{\alpha}{2} & 0 & 0 \\
0 & -\frac{\alpha}{2} & -2 \omega \\
0 & 2 \omega & 0
\end{array}\right),
$$

and the noise contribution

$$
\mathcal{L}_{\gamma}=\left(\begin{array}{ccc}
-2\left(\gamma_{22}+\gamma_{33}\right) & 2 \gamma_{12} & 2 \gamma_{13} \\
2 \gamma_{12} & -2\left(\gamma_{11}+\gamma_{33}\right) & 2 \gamma_{23} \\
2 \gamma_{13} & 2 \gamma_{23} & -2\left(\gamma_{11}+\gamma_{22}\right)
\end{array}\right) .
$$

Equation (12) is formally solved by exponentiating the Liouvillian:

$$
\boldsymbol{s}(t)=e^{\mathcal{L} t} \boldsymbol{s}(0)
$$

\section{QUANTUM ZENO EFFECT IN THE ABSENCE OF NOISE}

Before dealing with the effect of noise on the QZE, we review the features of the QZE in the absence of noise $[8,17,24$ 30]. These known results will serve as a benchmark against which to assess the effects of noise on the QZE. A convenient quantifier for this goal is the survival probability, i.e., the probability that the system would be found in its initial state $[|0\rangle$, cf. Eq. (2)], when measured projectively at time $t$. This is given by

$$
\mathcal{P}(t)=\operatorname{Tr}\left[\rho_{s}(t) \rho_{s}(0)\right]=\frac{1+z(t)}{2} .
$$

In the absence of noise, $\gamma_{i j}=0$, the evolution superoperator $\mathcal{L}$ in Eq. (12) reduces to

$$
\mathcal{L}^{(\overline{\mathrm{n}})}=\mathcal{L}_{0}=\left(\begin{array}{ccc}
-\frac{\alpha}{2} & 0 & 0 \\
0 & -\frac{\alpha}{2} & -2 \omega \\
0 & 2 \omega & 0
\end{array}\right),
$$

where the superscript $(\overline{\mathrm{n}})$ represents the absence of noise. The evolution of the Bloch vector $\boldsymbol{s}(t)$ is obtained explicitly by diagonalizing the Liouvillian, $\mathcal{L}^{(\overline{\mathrm{n}})}=W^{(\overline{\mathrm{n}})} \Lambda^{(\overline{\mathrm{n}})}\left(W^{(\overline{\mathrm{n}})}\right)^{-1}$. Here $\Lambda^{(\overline{\mathrm{n}})}$ is the diagonal matrix of eigenvalues of $\mathcal{L}^{(\overline{\mathrm{n}})}$, given by

$$
\begin{gathered}
\lambda_{1}^{(\overline{\mathrm{n}})}=-\frac{\alpha}{2}, \\
\lambda_{2}^{(\overline{\mathrm{n}})}=-\frac{1}{4}\left(\alpha+\sqrt{\alpha^{2}-64 \omega^{2}}\right), \\
\lambda_{3}^{(\overline{\mathrm{n}})}=-\frac{1}{4}\left(\alpha-\sqrt{\alpha^{2}-64 \omega^{2}}\right) .
\end{gathered}
$$

Then $s(t)=W^{(\overline{\mathrm{n}})} \exp \left(\Lambda^{(\overline{\mathrm{n}})} t\right)\left(W^{(\overline{\mathrm{n}})}\right)^{-1} s(0)$, and, consequently, the survival probability reads

$$
\begin{aligned}
\mathcal{P}^{(\overline{\mathrm{n}})}(t)=\frac{1}{2}[1 & +e^{-\frac{\alpha t}{4}}\left(\cosh \frac{t \sqrt{\alpha^{2}-64 \omega^{2}}}{4}\right. \\
& \left.\left.+\frac{\alpha}{\sqrt{\alpha^{2}-64 \omega^{2}}} \sinh \frac{t \sqrt{\alpha^{2}-64 \omega^{2}}}{4}\right)\right] .
\end{aligned}
$$

For any finite measurement strength $\alpha$, the probability asymptotically decays to $1 / 2$ at $t \rightarrow \infty$. However, the rate of decay depends on the measurement strength. In the limit of infinitely frequent projective measurements, $\alpha \rightarrow \infty$, the survival probability is equal to 1 at all times, so the system never leaves its initial state, which corresponds to the perfect QZE. For sufficiently weak measurements, $\alpha<8 \omega$, the survival probability exhibits not only decay, but also oscillations.

In the following sections, we analyze the effect of the Hamiltonian noise on the survival probability. We are specifically interested in three aspects of its behavior:

(i) The short-time behavior; at $t \rightarrow 0$,

$$
\mathcal{P}^{(\overline{\mathrm{n}})}(t \rightarrow 0)=1-\omega^{2} t^{2}+\frac{\alpha \omega^{2} t^{3}}{6}+O\left(t^{4}\right) .
$$

We see that, for any measurement strength, the probability initially decays quadratically in time. The presence of measurements increases the survival probability at short times; however, this is a cubic order effect. Note that, in this limit, $\alpha t \ll 1$ is implicitly assumed, hence the freezing of the state $\left(\mathcal{P}^{(\overline{\mathrm{n}})} \equiv 1\right)$ as $\alpha \rightarrow \infty$ is not apparent.

(ii) The oscillations of the survival probability. The survival probability oscillates as a function of time for $\alpha<8 \omega$, which is a consequence of the eigenvalues $\lambda_{2}^{(\bar{n})}$ and $\lambda_{3}^{(\bar{n})}$ being complex. The oscillations vanish for $\alpha \geqslant 8 \omega$ (cf. Fig. 2). The value of $\alpha=8 \omega=\alpha_{\mathrm{exc}}^{(\overline{\mathrm{n}})}$, where the Liouvillian's eigenvalues become degenerate, $\lambda_{2}^{(\overline{(})}=\lambda_{3}^{(\bar{n})}$, is an exceptional point of the evolution superoperator [45-47] and can be identified as the critical measurement strength for the onset of the QZE regime $[40,41]$. 


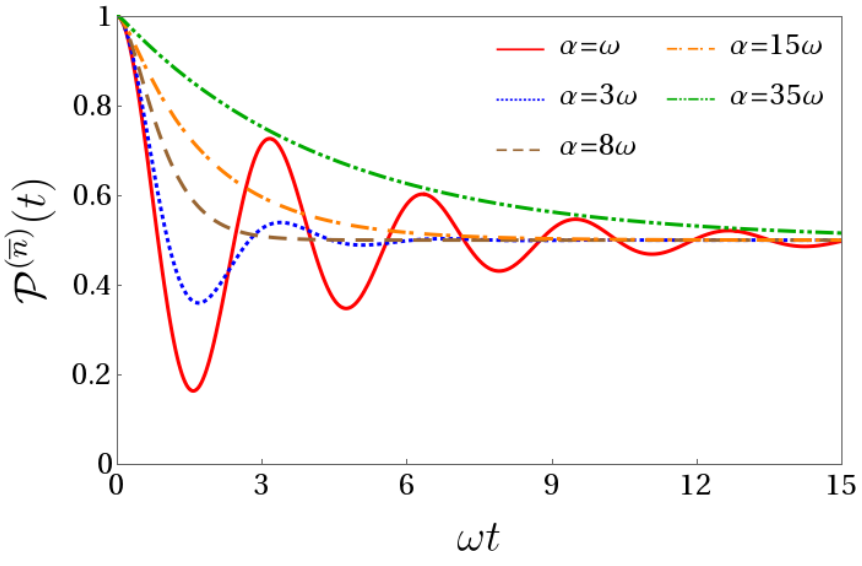

FIG. 2. The behavior of the survival probability $\mathcal{P}^{(\overline{\mathrm{n}})}(t)$ for different measurement strengths in the absence of noise. The survival probability has an oscillatory behavior for $\alpha<8 \omega$ and nonoscillatory behavior for $\alpha \geqslant 8 \omega$.

(iii) The long-time behavior. At $t \rightarrow \infty$, the behavior is determined by the slowest decaying eigenstate of the evolution superoperator, which is the one associated with eigenvalue $\lambda_{3}^{(\bar{n})}$. Thus, at long times, the survival probability can be written as

$$
\mathcal{P}^{(\overline{\mathrm{n}})}(t \rightarrow \infty)=\frac{1}{2}+e^{-t\left|\operatorname{Re} \lambda_{3}^{(\overline{\mathrm{n}})}\right|} f(t),
$$

where $f(t)$ is either a constant or a bounded oscillating function. Note that the decay rate $\left|\operatorname{Re} \lambda_{3}^{(\overline{\mathrm{n}})}\right|$, for a fixed $\omega$, exhibits a maximum at $\alpha=8 \omega=\alpha_{\mathrm{exc}}^{(\overline{\mathrm{n}})}$, as shown in Fig. 3. That is, the survival probability long-time decay rate decreases with increasing $\alpha$ at $\alpha>8 \omega$, while the behavior is the opposite at $\alpha<8 \omega$.

Therefore, the effect of the Hamiltonian noise on the QZE can be assessed by three quantifiers: (i) the amount of suppression of the survival probability at short times, (ii) the shift of the exceptional point, and (iii) the effect on the survival probability's long-time decay rate.

\section{EFFECT OF NOISE ON QUANTUM ZENO EFFECT}

We are now in a position to analyze the effect of Hamiltonian noise on the QZE. This is generally described by six

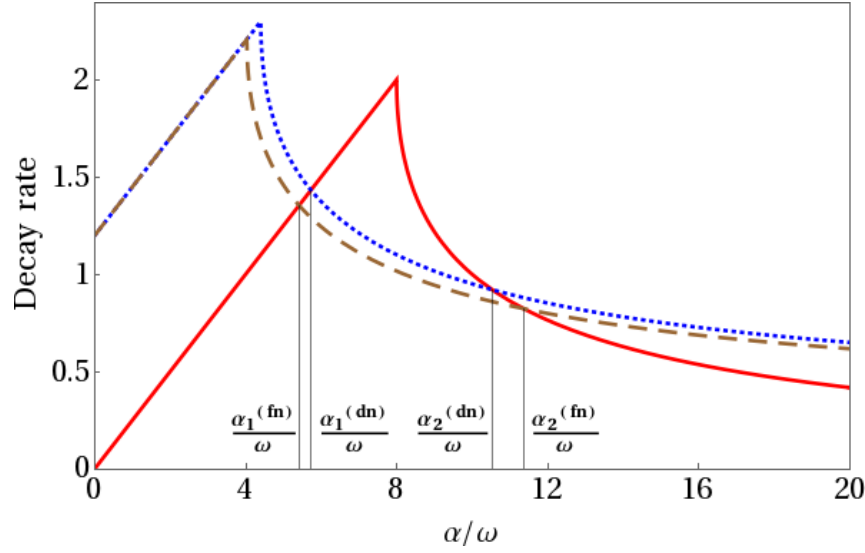

FIG. 3. The behavior of the long-time decay rate of the survival probability as a function of the measurement strength $\alpha$-in the absence of noise (solid red), when only the diagonal noise $\gamma_{i i}$ is present (dotted blue), and in the presence of both the diagonal and off-diagonal noise (dashed brown). The decay rates are maximum at the respective exceptional points. It can be seen that, depending on the measurement strength, noise can enhance or suppress the decay rate. The presence of off-diagonal noise component increases the interval over which the decay rate is suppressed compared with the case of diagonal noise. The solid vertical lines mark the measurement strengths that define the interval over which the decay rate is suppressed by the noise, cf. Eqs. (32)-(33) and (41)-(42). The plots are obtained for $\gamma_{11}=0.05 \omega, \gamma_{22}=0.1 \omega, \gamma_{12}=\gamma_{13}=0, \gamma_{23}=0.3 \omega$, and $\gamma_{33}=\omega$.

independent parameters $\left\{\gamma_{i j}\right\}$ with $i \leqslant j$, cf. Eq. (14). We start by considering the case of diagonal noise $\gamma_{i j} \propto \delta_{i j}$, which demonstrates all the qualitative features appearing due to noise, see Sec. IV A. In Sec. IV B, we include the off-diagonal terms and discuss their effect.

\section{A. Diagonal noise}

The diagonal noise is defined by $\gamma_{i j}=\gamma_{i i} \delta_{i j}$ with $\gamma_{i i} \geqslant 0$. Physically, this may originate from the fluctuations of the energy difference between $|0\rangle$ and $|1\rangle$ states $\left(\gamma_{33} \neq 0\right)$, the fluctuations of the Rabi frequency $\omega\left(\gamma_{11} \neq 0\right)$, or the fluctuations of the Hamiltonian direction in the $x y$ plane $\left(\gamma_{11} \neq 0\right.$ and $\gamma_{22} \neq 0$ ). The Liouvillian $\mathcal{L}$ [Eq. (12)] then takes the form

$$
\mathcal{L}^{(\mathrm{dn})}=\mathcal{L}_{0}+\mathcal{L}_{\gamma}=\left(\begin{array}{ccc}
-\frac{\alpha+4\left(\gamma_{22}+\gamma_{33}\right)}{2} & 0 & 0 \\
0 & -\frac{\alpha+4\left(\gamma_{11}+\gamma_{33}\right)}{2} & -2 \omega \\
0 & 2 \omega & -2\left(\gamma_{11}+\gamma_{22}\right)
\end{array}\right),
$$

with the corresponding eigenvalues

$$
\begin{gathered}
\lambda_{1}^{(\mathrm{dn})}=-\frac{\alpha_{\mathrm{dn}}}{2}-4 \gamma_{22}, \\
\lambda_{2}^{(\mathrm{dn})}=-\frac{1}{4}\left(\alpha_{\mathrm{dn}}+8\left(\gamma_{11}+\gamma_{22}\right)+\sqrt{\alpha_{\mathrm{dn}}^{2}-64 \omega^{2}}\right), \\
\lambda_{3}^{(\mathrm{dn})}=-\frac{1}{4}\left(\alpha_{\mathrm{dn}}+8\left(\gamma_{11}+\gamma_{22}\right)-\sqrt{\alpha_{\mathrm{dn}}^{2}-64 \omega^{2}}\right) .
\end{gathered}
$$


Here $\alpha_{\mathrm{dn}}=\alpha-4\left(\gamma_{22}-\gamma_{33}\right)$ plays the role of the renormalized measurement strength in some of the observables (see below). With the system's initial state corresponding to $|0\rangle$, cf. Eq. (2), one obtains the survival probability

$$
\mathcal{P}^{(\mathrm{dn})}(t)=\frac{1}{2}\left[1+e^{-\frac{t\left[\alpha_{\mathrm{dn}}+8\left(\gamma_{1}+\gamma_{22}\right)\right]}{4}}\left(\cosh \frac{t \sqrt{\alpha_{\mathrm{dn}}^{2}-64 \omega^{2}}}{4}+\frac{\alpha_{\mathrm{dn}}}{\sqrt{\alpha_{\mathrm{dn}}^{2}-64 \omega^{2}}} \sinh \frac{t \sqrt{\alpha_{\mathrm{dn}}^{2}-64 \omega^{2}}}{4}\right)\right] .
$$

In the limit $\alpha \rightarrow \infty, \mathcal{P}^{(\mathrm{dn})}(t)=\frac{1}{2}\left(1+e^{-2\left(\gamma_{11}+\gamma_{22}\right) t}\right)$. Thence, the noise will generically prevent a full freezing of the state with $\mathcal{P}^{(\mathrm{dn})}(t) \rightarrow 1$ in the ideal strong measurement case, as was previously pointed out in Ref. [39]. However, for realistic finite values of the measurement strength, the presence of noise can alter the survival probability behavior and the critical measurement strength both favorably and not favorably. First, it is evident from Eq. (24) that $\gamma_{33}$ acts solely to renormalize the measurement strength $\alpha$ (in agreement with the results of Refs. [36,37]). Therefore, the noise along the $z$ axis enhances the effective measurement strength and thence the QZE. This has an intuitive explanation: A nonzero $\sigma_{z}$ term in the Hamiltonian would reduce the size of Rabi oscillations enabled by the $\omega \sigma_{x}$ term, thus enhancing the survival probability; $\gamma_{33} \neq 0$ corresponds to having a fluctuating $\sigma_{z}$ term in the Hamiltonian that similarly enhances the survival probability. The effect of $\gamma_{11}$ is also rather clear: it induces dephasing between $\sigma_{x}$ eigenstates, hence inducing an exponential decay of the $z$ component of the Bloch vector. This counteracts the onset of the QZE, and in fact it prevents the full freezing of the state for $\alpha \rightarrow \infty$. The noise along the $y$ axis has the opposite effect of $\gamma_{33}$ in renormalizing the measurement strength and acts analogously to $\gamma_{11}$ in affecting the exponential decay, so to counteract overall the QZE.

Focusing specifically on the quantifiers of the QZE introduced in Sec. III, the short-time behavior of the survival probability is given by

$$
\begin{aligned}
\mathcal{P}^{(\mathrm{dn})}(t)= & 1-\left(\gamma_{11}+\gamma_{22}\right) t-\left[\omega^{2}-\left(\gamma_{11}+\gamma_{22}\right)^{2}\right] t^{2} \\
& +\frac{1}{6}\left(\omega^{2} \alpha-4\left(\gamma_{11}+\gamma_{22}\right)^{3}+4 \omega^{2}\left(3 \gamma_{11}+2 \gamma_{22}+\gamma_{33}\right)\right) t^{3}+O\left(t^{4}\right) .
\end{aligned}
$$

Comparing this to the noiseless case in Eq. (22), we see that the presence of noise induces a linear-in-time decay. Therefore, at short times, the QZE is suppressed by noise unless $\gamma_{11}=\gamma_{22}=0$, see Fig. 4. If this condition is satisfied, then the short-time behavior is equivalent to that in the noiseless case with a renormalized measurement strength, $\alpha \rightarrow \alpha+4 \gamma_{33}>\alpha$.

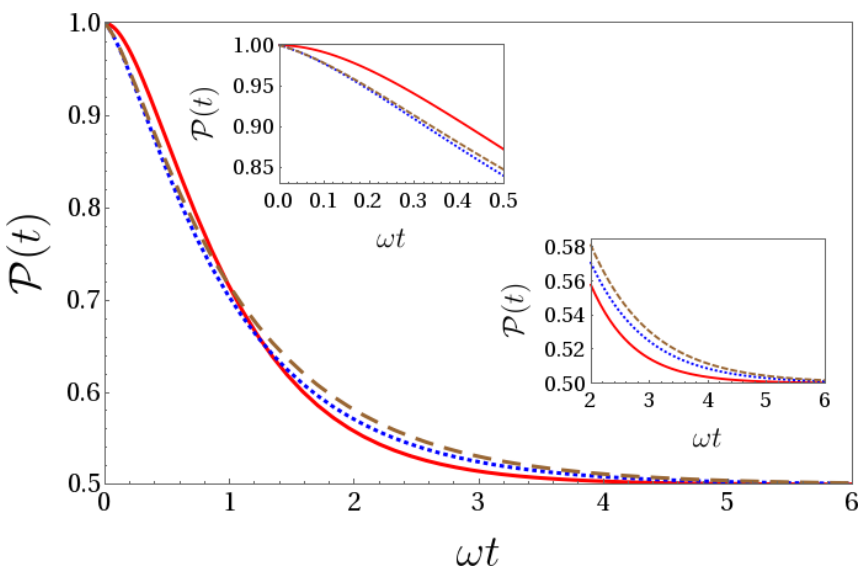

FIG. 4. Time dependence of the survival probability $\mathcal{P}(t)$, cf. Eq. (16), for the cases of no noise $\left(\gamma_{i j}=0\right.$, solid red), diagonal noise ( $\gamma_{11}=0.05 \omega, \gamma_{22}=0.1 \omega, \gamma_{33}=\omega$, dotted blue), and full noise (as diagonal noise plus $\gamma_{23}=0.3 \omega$, dashed brown). The insets focus on the short-time and long-time behavior. The noise always decreases the survival probability at short times, however, at long times it may actually increase it (the parameters used in the plot correspond to the latter scenario). The plots are obtained for $\alpha=8.5 \omega$. The enhancement of the survival probability at long times can be more significant; however, this only happens for $\gamma_{33}$-dominated noise $\left(\gamma_{33} / \gamma_{11}, \gamma_{33} / \gamma_{22} \gtrsim 100\right)$.
Therefore, noise along the $z$ axis alone does enhance the QZE at short times.

The analysis of the oscillatory dynamics of the survival probability, controlled by the exceptional point of the Liouvillian spectrum, reveals a rather different picture. The exceptional point (where $\lambda_{2}^{(\mathrm{dn})}=\lambda_{3}^{(\mathrm{dn})}$ ) now occurs at

$$
\alpha_{\mathrm{dn}}=8 \omega \Longleftrightarrow \alpha=8 \omega+4\left(\gamma_{22}-\gamma_{33}\right)=\alpha_{\mathrm{exc}}^{(\mathrm{dn})} .
$$

This implies that, for a given $\omega$, the onset of the QZE regime happens at a smaller measurement strength as long as $\gamma_{33}>$ $\gamma_{22}$, independently of $\gamma_{11}$. This condition for enhancing the QZE is drastically different from the one found when considering the short-time dynamics.

Finally, consider the long-time decay rate of the survival probability. The decay rate is given by $\left|\operatorname{Re} \lambda_{3}^{(\mathrm{dn})}\right|$, to be compared with the decay rate in the absence of noise, $\left|\operatorname{Re} \lambda_{3}^{(\bar{n})}\right|$, cf. Eqs. (27) and (20), respectively. We find that, generically, the noise increases the decay rate, except when the following three conditions are satisfied simultaneously: (a) $\gamma_{33}>\gamma_{22}$, (b) $\omega>\left(\gamma_{11}+\gamma_{22}\right)\left(\gamma_{11}+\gamma_{33}\right) /\left(\gamma_{33}-\gamma_{22}\right)$, (c) the measurement strength $\alpha$ falls in the interval

$$
\alpha_{1}^{(\mathrm{dn})}<\alpha<\alpha_{2}^{(\mathrm{dn})},
$$

with

$$
\begin{aligned}
\alpha_{1}^{(\mathrm{dn})}=4\left(\gamma_{22}\right. & \left.-\gamma_{33}+\sqrt{4 \omega^{2}+\left(2 \gamma_{11}+\gamma_{22}+\gamma_{33}\right)^{2}}\right), \\
\alpha_{2}^{(\mathrm{dn})}= & 2\left(\gamma_{22}-\gamma_{33}+\left(2 \gamma_{11}+\gamma_{22}+\gamma_{33}\right)\right. \\
& \times \sqrt{\left.1+\frac{4 \omega^{2}}{\left(\gamma_{11}+\gamma_{22}\right)\left(\gamma_{11}+\gamma_{33}\right)}\right)} .
\end{aligned}
$$


Such suppression of the decay rate (enhancement of the longtime survival probability) is illustrated in Figs. 3 and 4.

We thus see that the conditions for enhancing the QZE by the noise differ depending on the quantity under consideration. The short-time survival probability is almost always suppressed by noise. The onset of the QZE regime (i.e., the exceptional point) happens at a lower measurement strength when $\gamma_{33}>\gamma_{22}$. The enhancement of the long-time survival probability agrees with that obtained from the exceptional point shift in the requirement of $\gamma_{33}>\gamma_{22}$. At the same time, the long-time survival probability enhancement poses extra conditions: the noiseless Hamiltonian should be sufficiently strong compared with some noise-related quantity (b), and the measurement strength should belong to an interval close to the exceptional point (c).

\section{B. Generic noise}

We now analyze the case of generic noise with all $\gamma_{i j} \neq 0$. We focus on the regime of sufficiently weak noise, $\left|\gamma_{i j}\right| \ll$ $\alpha, \omega$. When $\alpha \rightarrow \infty$, the $z$ component of the Bloch vector is effectively decoupled from the $x$ and $y$ components, cf. Eqs. (12)-(14), implying that the effect of the off-diagonal noise components onto the survival probability can be neglected. Therefore, we focus on sufficiently small $\alpha$. For sufficiently small $\alpha$, one can compute the Liouvillian eigenvalues perturbatively in $\gamma_{12} /\left|\lambda_{1}^{(\bar{n})}-\lambda_{2}^{(\bar{n})}\right| \ll 1$ and $\gamma_{13} / \mid \lambda_{1}^{(\bar{n})}-$ $\lambda_{3}^{(\bar{n})} \mid \ll 1$ and show that these parameters only contribute at the second order of perturbation theory. Thus, the effect of cross correlations between the noise along the $x$ and the other two axes is negligibly small in the considered regime.

Diagonalizing the evolution superoperator (12) and neglecting the contributions of $\gamma_{12}$ and $\gamma_{13}$, we obtain

$$
\begin{gathered}
\lambda_{1}^{(\mathrm{fn})}=-\frac{\alpha_{\mathrm{dn}}}{2}-4 \gamma_{22}, \\
\lambda_{2}^{(\mathrm{fn})}=-\frac{1}{4}\left(\alpha_{\mathrm{dn}}+8\left(\gamma_{11}+\gamma_{22}\right)+\sqrt{\alpha_{\mathrm{dn}}^{2}-64\left(\omega^{2}-\gamma_{23}^{2}\right)}\right), \\
\lambda_{3}^{(\mathrm{fn})}=-\frac{1}{4}\left(\alpha_{\mathrm{dn}}+8\left(\gamma_{11}+\gamma_{22}\right)-\sqrt{\alpha_{\mathrm{dn}}^{2}-64\left(\omega^{2}-\gamma_{23}^{2}\right)}\right) .
\end{gathered}
$$

Note that, if $\gamma_{12}=\gamma_{13}=0$, our analysis is exact and is valid for arbitrary-strength noise.

The survival probability is then obtained as

$$
\mathcal{P}^{(\mathrm{fn})}=\frac{1}{2}\left[1+e^{-\frac{t\left(\alpha_{\mathrm{dn}}+8\left(\gamma_{11}+\gamma_{22}\right)\right)}{4}}\left(\cosh \frac{t \sqrt{\alpha_{\mathrm{dn}}^{2}-64\left(\omega^{2}-\gamma_{23}^{2}\right)}}{4}+\frac{\alpha_{\mathrm{dn}}}{\sqrt{\alpha_{\mathrm{dn}}^{2}-64\left(\omega^{2}-\gamma_{23}^{2}\right)}} \sinh \frac{t \sqrt{\alpha_{\mathrm{dn}}^{2}-64\left(\omega^{2}-\gamma_{23}^{2}\right)}}{4}\right)\right] .
$$

The short-time behavior of the survival probability is thus the same as in the case of diagonal noise (29), modulo replacing $\omega^{2} \rightarrow \omega^{2}-\gamma_{23}^{2}$,

$$
\begin{aligned}
\mathcal{P}^{(\mathrm{fn})}= & 1-\left(\gamma_{11}+\gamma_{22}\right) t-\left[\omega^{2}-\gamma_{23}^{2}-\left(\gamma_{11}+\gamma_{22}\right)^{2}\right] t^{2} \\
& +\frac{1}{6}\left\{\left(\omega^{2}-\gamma_{23}^{2}\right)\left[\alpha+4\left(3 \gamma_{11}+2 \gamma_{22}+\gamma_{33}\right)\right]-4\left(\gamma_{11}+\gamma_{22}\right)^{3}\right\} t^{3}+O\left(t^{4}\right) .
\end{aligned}
$$

Hence, the short-time survival probability is always reduced by the noise unless $\gamma_{11}=\gamma_{22}=0$ (note that the requirement of $\gamma_{i j}$ being a positive-semidefinite matrix implies that, in this case, $\gamma_{i j}=0$ unless $i=j=3$ ). Note also the subleading $t^{2}$ term, where the presence of $\gamma_{23}$ enhances the survival probability, see Fig. 4.

The exceptional point happens when

$$
\alpha=4\left(2 \sqrt{\omega^{2}-\gamma_{23}^{2}}+\gamma_{22}-\gamma_{33}\right)=\alpha_{\mathrm{exc}}^{(\mathrm{fn})} .
$$

This is a smaller value of $\alpha$ than $\alpha_{\mathrm{exc}}^{(\overline{\mathrm{n}})}=8 \omega$ provided that $\gamma_{33}>\gamma_{22}$, or if $\gamma_{33}<\gamma_{22}$ and $4 \omega<\left(\gamma_{22}-\gamma_{33}\right)+4 \gamma_{23}^{2} /\left(\gamma_{22}-\gamma_{33}\right)$. When $\left|\gamma_{i j}\right| \ll \omega$, the last condition on $\omega$ can only be satisfied when $\gamma_{22}-\gamma_{33} \ll\left|\gamma_{23}\right|$.

Finally, for the effect of noise in the long-time limit, we compare $\left|\operatorname{Re} \lambda_{3}^{(\bar{n})}\right|$ and $\left|\operatorname{Re} \lambda_{3}^{(\mathrm{fn})}\right|$, cf. Eqs. (20) and (36). Similarly to the case of diagonal noise, the long-time decay rate is reduced if and only if the following three conditions are satisfied: (a) $\gamma_{33}>\gamma_{22}$, (b) $\omega>\left[\left(\gamma_{11}+\gamma_{22}\right)\left(\gamma_{11}+\gamma_{33}\right)-\gamma_{23}^{2}\right] /\left(\gamma_{33}-\gamma_{22}\right)$, (c) the measurement strength belongs to the interval

$$
\alpha_{1}^{(\mathrm{fn})}<\alpha<\alpha_{2}^{(\mathrm{fn})}
$$

with

$$
\begin{gathered}
\alpha_{1}^{(\mathrm{fn})}=4\left(\gamma_{22}-\gamma_{33}+\sqrt{4\left(\omega^{2}-\gamma_{23}^{2}\right)+\left(2 \gamma_{11}+\gamma_{22}+\gamma_{33}\right)^{2}}\right), \\
\alpha_{2}^{(\mathrm{fn})}=2\left[\left(\gamma_{22}-\gamma_{33}\right)\left(1-\frac{\gamma_{23}^{2}}{\left(\gamma_{11}+\gamma_{22}\right)\left(\gamma_{11}+\gamma_{33}\right)}\right)\right. \\
\left.+\left(2 \gamma_{11}+\gamma_{22}+\gamma_{33}\right) \sqrt{\left(1-\frac{\gamma_{23}^{2}}{\left(\gamma_{11}+\gamma_{22}\right)\left(\gamma_{11}+\gamma_{33}\right)}\right)^{2}+\frac{4 \omega^{2}}{\left(\gamma_{11}+\gamma_{22}\right)\left(\gamma_{11}+\gamma_{33}\right)}}\right] .
\end{gathered}
$$


Note that the off-diagonal noise enhances the QZE under a wider range of conditions. Indeed, when $\gamma_{23} \neq 0$, the restriction on $\omega$ is weaker than for the diagonal noise with the same $\gamma_{i i}$. Furthermore, for $\omega>\left(\gamma_{11}+\gamma_{22}\right)\left(\gamma_{11}+\gamma_{33}\right) /\left(\gamma_{33}-\gamma_{22}\right)$ at which the diagonal noise allows for reducing the decay rate, adding the off-diagonal component increases the relevant interval of measurement strengths: $\alpha_{1}^{(\mathrm{fn})}<\alpha_{1}^{(\mathrm{dn})}$ and $\alpha_{2}^{(\mathrm{fn})}>$ $\alpha_{2}^{(\mathrm{dn})}$, as illustrated in Fig. 3.

We thus see that the off-diagonal noise components do not qualitatively change the effect of noise on the QZE. It is interesting to note, though, that correlations between the $y$ and the $z$ components of noise $\left(\gamma_{23} \neq 0\right)$ tend to enhance the QZE compared with the purely diagonal noise.

\section{CONCLUSION}

In this work we have investigated the effect of noise in the system Hamiltonian on the QZE behavior induced by continuous partial measurement. We have found a quite rich behavior: the effect is significantly different when looking at different QZE quantifiers. Specifically, we have investigated the effect of noise on the short-time and long-time survival probabilities and on the critical measurement strength determining the onset of the QZE regime. We have found that the short-time survival probability is essentially always reduced by noise, except for very-fine-tuned conditions, namely, for a fluctuating term that commutes with the measured observable. The onset of the QZE can be shifted towards larger or smaller measurement strengths depending on the details of the noise and the averaged Hamiltonian parameters. Finally, the longtime survival probability can also be increased or decreased, yet this is not determined by the properties of the noise only. The same noise can enhance or suppress the long-time survival probability depending on the measurement strength and the noiseless part of the system's Hamiltonian. Notably, the conditions for a shift of the QZE onset towards a lower measurement strength differ from those required to enhance the long time survival probability. Our results can be relevant for QZE-based protocols in systems subject to fluctuations, e.g., to optimize working points in parameter space in order to enhance the desired features of the QZE.

In this work we focused on Hermitian Hamiltonians. The interplay of non-Hermitian Hamiltonian noise [48] with measurement dynamics may be of interest for applications in non-Hermitian systems.

\section{ACKNOWLEDGMENTS}

P.K. and K.S. acknowledge funding by the Deutsche Forschungsgemeinschaft (DFG, German Research Foundation)_-Projektnummer 277101999_-TRR 183 (project C01) and Projektnummer EG 96/13-1, and by the Israel Science Foundation (ISF). A.R. acknowledges the EPSRC via Grant No. EP/P010180/1.
[1] B. Misra and E. C. G. Sudarshan, The Zeno's paradox in quantum theory, J. Math. Phys. 18, 756 (1977).

[2] W. M. Itano, D. J. Heinzen, J. J. Bollinger, and D. J. Wineland, Quantum Zeno effect, Phys. Rev. A 41, 2295 (1990).

[3] P. Facchi and S. Pascazio, Quantum Zeno dynamics: Mathematical and physical aspects, J. Phys. A: Math. Theor. 41, 493001 (2008).

[4] D. Home and M. A. B. Whitaker, A Conceptual analysis of quantum Zeno; Paradox, measurement, and experiment, Ann. Phys. (NY) 258, 237 (1997).

[5] H. Nakazato, M. Namiki, and S. Pascazio, Temporal behavior of quantum mechanical systems, Int. J. Mod. Phys. B 10, 247 (1996).

[6] P. Kwiat, H. Weinfurter, T. Herzog, A. Zeilinger, and M. A. Kasevich, Interaction-Free Measurement, Phys. Rev. Lett. 74, 4763 (1995).

[7] M. C. Fischer, B. Gutiérrez-Medina, and M. G. Raizen, Observation of the Quantum Zeno and Anti-Zeno Effects in an Unstable System, Phys. Rev. Lett. 87, 040402 (2001).

[8] E. W. Streed, J. Mun, M. Boyd, G. K. Campbell, P. Medley, W. Ketterle, and D. E. Pritchard, Continuous and Pulsed Quantum Zeno Effect, Phys. Rev. Lett. 97, 260402 (2006).

[9] P.-W. Chen, D.-B. Tsai, and P. Bennett, Quantum Zeno and antiZeno effect of a nanomechanical resonator measured by a point contact, Phys. Rev. B 81, 115307 (2010).

[10] D. H. Slichter, C. Müller, R. Vijay, S. J. Weber, A. Blais, and I. Siddiqi, Quantum Zeno effect in the strong measurement regime of circuit quantum electrodynamics, New J. Phys. 18, 053031 (2016).

[11] P. Facchi and S. Pascazio, Quantum Zeno Subspaces, Phys. Rev. Lett. 89, 080401 (2002).

[12] F. Schäfer, I. Herrera, S. Cherukattil, C. Lovecchio, F. S. Cataliotti, F. Caruso, and A. Smerzi, Experimental realization of quantum zeno dynamics, Nat. Commun. 5, 3194 (2014).

[13] A. Signoles, A. Facon, D. Grosso, I. Dotsenko, S. Haroche, J.-M. Raimond, M. Brune, and S. Gleyzes, Confined quantum Zeno dynamics of a watched atomic arrow, Nat. Phys. 10, 715 (2014).

[14] A. Beige, D. Braun, B. Tregenna, and P. L. Knight, Quantum Computing Using Dissipation to Remain in a Decoherence-Free Subspace, Phys. Rev. Lett. 85, 1762 (2000).

[15] S. Maniscalco, F. Francica, R. L. Zaffino, N. Lo Gullo, and F. Plastina, Protecting Entanglement via the Quantum Zeno Effect, Phys. Rev. Lett. 100, 090503 (2008).

[16] Y.-S. Kim, J.-C. Lee, O. Kwon, and Y.-H. Kim, Protecting entanglement from decoherence using weak measurement and quantum measurement reversal, Nat. Phys. 8, 117 (2012).

[17] G. A. Paz-Silva, A. T. Rezakhani, J. M. Dominy, and D. A. Lidar, Zeno Effect for Quantum Computation and Control, Phys. Rev. Lett. 108, 080501 (2012).

[18] Y.-H. Chen and T. A. Brun, Continuous quantum error detection and suppression with pairwise local interactions, Phys. Rev. Research 2, 043093 (2020).

[19] S. Patsch, S. Maniscalco, and C. P. Koch, Simulation of openquantum-system dynamics using the quantum Zeno effect, Phys. Rev. Research 2, 023133 (2020). 
[20] F. Tonielli, N. Chakraborty, F. Grusdt, and J. Marino, Ramsey interferometry of non-Hermitian quantum impurities, Phys. Rev. Research 2, 032003 (2020).

[21] P. E. Dolgirev, J. Marino, D. Sels, and E. Demler, Non-Gaussian correlations imprinted by local dephasing in fermionic wires, Phys. Rev. B 102, 100301(R) (2020).

[22] A. I. Shushin, The effect of measurements, randomly distributed in time, on quantum systems: Stochastic quantum Zeno effect, J. Phys. A: Math. Theor. 44, 055303 (2011).

[23] S. Gherardini, S. Gupta, F. S. Cataliotti, A. Smerzi, F. Caruso, and S. Ruffo, Stochastic quantum Zeno by large deviation theory, New J. Phys. 18, 013048 (2016).

[24] J. Gong and S. A. Rice, Measurement-assisted coherent control, J. Chem. Phys. 120, 9984 (2004).

[25] K. Koshino and A. Shimizu, Quantum Zeno effect by general measurements, Phys. Rep. 412, 191 (2005).

[26] L. Xiao and J. A. Jones, NMR analogues of the quantum Zeno effect, Phys. Lett. A 359, 424 (2006).

[27] R. Ruskov, A. N. Korotkov, and A. Mizel, Quantum Zeno stabilization in weak continuous measurement of two qubits, Phys. Rev. B 73, 085317 (2006).

[28] H.-P. Breuer and F. Petruccione, The Theory of Open Quantum Systems (Oxford University Press, New York, 2007).

[29] D. Layden, E. Martín-Martínez, and A. Kempf, Perfect Zeno-like effect through imperfect measurements at a finite frequency, Phys. Rev. A 91, 022106 (2015).

[30] M. Zhang, C. Wu, Y. Xie, W. Wu, and P. Chen, Quantum Zeno effect by incomplete measurements, Quantum Inf. Process. 18, 97 (2019).

[31] A. C. Elitzur and S. Dolev, Nonlocal effects of partial measurements and quantum erasure, Phys. Rev. A 63, 062109 (2001).

[32] G. S. Paraoanu, Interaction-Free Measurements with Superconducting Qubits, Phys. Rev. Lett. 97, 180406 (2006).

[33] R. Ruskov, A. Mizel, and A. N. Korotkov, Crossover of phase qubit dynamics in the presence of a negative-result weak measurement, Phys. Rev. B 75, 220501(R) (2007).

[34] X.-Y. Xu, J.-S. Xu, C.-F. Li, Y. Zou, and G.-C. Guo, Experimental demonstration of nonlocal effects in the partial-collapse measurement and reversal process, Phys. Rev. A 83, 010101(R) (2011).
[35] M. S. Blok, C. Bonato, M. L. Markham, D. J. Twitchen, V. V. Dobrovitski, and R. Hanson, Manipulating a qubit through the backaction of sequential partial measurements and real-time feedback, Nat. Phys. 10, 189 (2014).

[36] H. Nakazato and S. Pascazio, Two-level system with a noisy Hamiltonian, J. Supercond. 12, 843 (1999).

[37] A. G. Kofman and G. Kurizki, Decay control in dissipative quantum systems, Acta Physica Slovaca 49, 541 (1999).

[38] A. G. Kofman, G. Kurizki, and T. Opatrný, Zeno and anti-Zeno effects for photon polarization dephasing, Phys. Rev. A 63, 042108 (2001).

[39] S. A. Gurvitz, L. Fedichkin, D. Mozyrsky, and G. P. Berman, Relaxation and the Zeno Effect in Qubit Measurements, Phys. Rev. Lett. 91, 066801 (2003).

[40] F. Li, J. Ren, and N. A. Sinitsyn, Quantum Zeno effect as a topological phase transition in full counting statistics and spin noise spectroscopy, Europhys. Lett. 105, 27001 (2014).

[41] K. Snizhko, P. Kumar, and A. Romito, Quantum Zeno effect appears in stages, Phys. Rev. Research 2, 033512 (2020).

[42] Z. K. Minev, S. O. Mundhada, S. Shankar, P. Reinhold, R. Gutiérrez-Jáuregui, R. J. Schoelkopf, M. Mirrahimi, H. J. Carmichael, and M. H. Devoret, To catch and reverse a quantum jump mid-flight, Nature (London) 570, 200 (2019).

[43] M. A. Nielsen and I. L. Chuang, Quantum Computation and Quantum Information (Cambridge University Press, Cambridge, 2010).

[44] H. M. Wiseman and G. J. Milburn, Quantum Measurement and Control (Cambridge University Press, 2010), p. 460.

[45] N. Hatano, Exceptional points of the Lindblad operator of a two-level system, Mol. Phys. 117, 2121 (2019).

[46] F. Minganti, A. Miranowicz, R. W. Chhajlany, and F. Nori, Quantum exceptional points of non-Hermitian Hamiltonians and Liouvillians: The effects of quantum jumps, Phys. Rev. A 100, 062131 (2019).

[47] F. Minganti, A. Miranowicz, R. W. Chhajlany, I. I. Arkhipov, and F. Nori, Hybrid-Liouvillian formalism connecting exceptional points of non-Hermitian Hamiltonians and Liouvillians via postselection of quantum trajectories, Phys. Rev. A 101, 062112 (2020).

[48] D. Burgarth, P. Facchi, G. Garnero, H. Nakazato, S. Pascazio, and K. Yuasa, Can decay be ascribed to classical noise? Open Sys. Info. Dyn. 24, 1750001 (2017). 